\title{
Capacitación y adiestramiento
}

\section{Development and Training}

\author{
César Uriel Ruiz Rosas
}

\begin{abstract}
:
Ergonomics is responsible for the study of the worker and work, is the set of scientific knowledge relating to man, which are necessary for the correct use of machines and devices, in order to obtain maximum efficiency, safety and compliance of them, the relationship that exists between ergonomics with training and training is due to the fact that one of the objectives of ergonomics is to take care of the wellbeing of the personnel, through Identifying, analyzing and reducing occupational hazards. This is where the training and training comes in. To know if any person is suitable or inept for the work that is proposed to be carried out, it is advisable that the staff know previously what this work consists of and if it is a position to be performed, to know which functions have been assigned to it. This position, for this reason is of the utmost importance that within organizations there is adequate training that guarantees not only the productivity of the organization, but also the development of human resources with an adequate work environment that guarantees such stability of the staff, avoiding inconveniences are already insecure acts because of a lack of inadequate training of the workers.
\end{abstract}

\section{Keywords:}

Ergonomics, Training, Worker, Work, Safety Industry

\section{Resumen:}

La ergonomía se encarga del estudio del trabajador y el trabajo, es el conjunto de conocimientos científicos relativos al hombre, que son necesarios para el uso correcto de máquinas y dispositivos, con el fin de obtener una máxima eficiencia, seguridad y conformidad de ellos, la relación que existe entre la ergonomía con la capacitación y el adiestramiento se debe a que uno de los objetivos de la ergonomía es cuidar el bienestar del personal, a través de Identificar, analizar y reducir los riesgos laborales es ahí donde entra la capacitación y el adiestramiento. Para saber si cualquier persona es apta o inepta para el trabajo que se propone realizar, es conveniente que el personal conozca previamente en qué consiste dicho trabajo y si se trata de un puesto a desempeñar, saber cuáles son las funciones que se le han asignado a dicho puesto, por tal motivo es de suma importancia que dentro de las organizaciones exista una adecuada capacitación que garantice no solo la productividad de la organización, sino también el desarrollo del recurso humano con un ambiente de trabajo adecuado que garantice dicho estabilidad del personal, evitando inconvenientes ya sean actos inseguros como consecuencia de una falta o inadecuada capacitación del trabajador

\section{Palabras Clave:}

Ergonomía, Capacitación, Adiestramiento, Trabajador, Trabajo, Seguridad Industria

Universidad Autónoma del Estado de Hidalgo. Escuela Superior Tepeji del Río, Av. del maestro, 41, Col.

Noxtongo, Tepeji del Río de Ocampo, Hidalgo, México. +52 (771) 717 2000, Ext.: 5850, 5851 


\section{CAPACITACION Y ADISESTRAMIENTO
ENFOCADO A LA ERGONOMIA}

¿Qué es la Ergonomía?
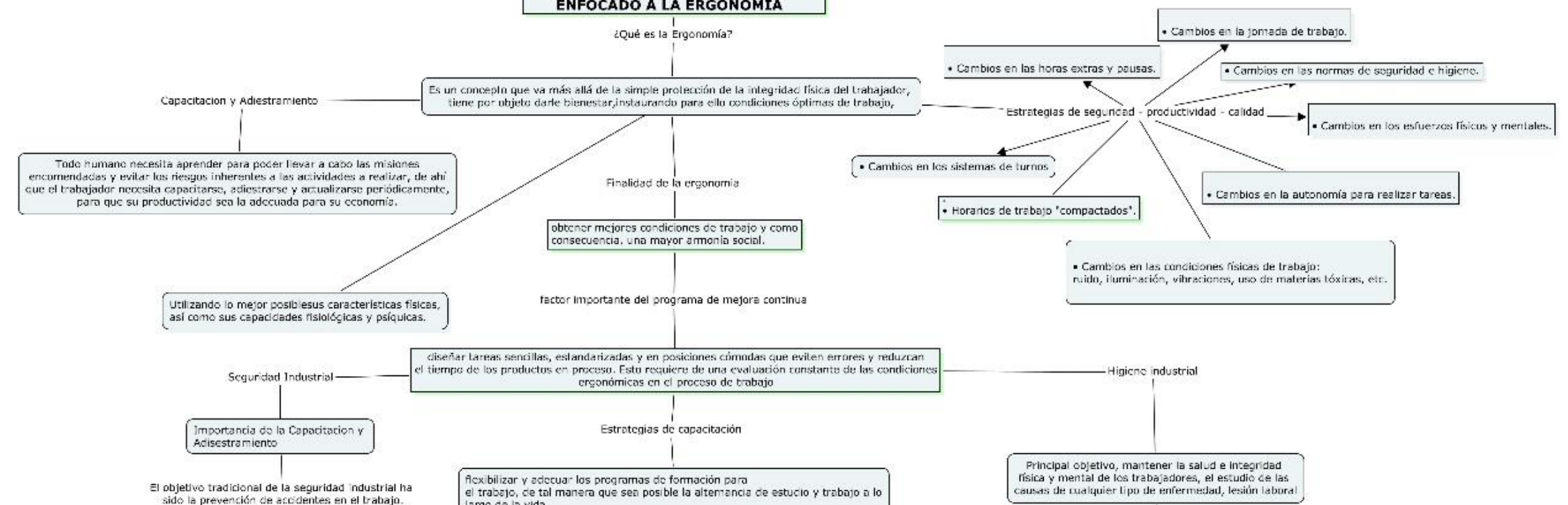

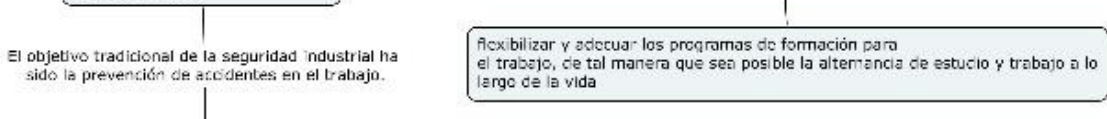

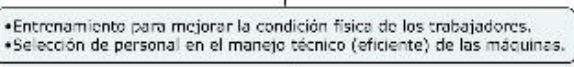

$\frac{5 \text { Se retlere exclusivamente a a persona }}{1}$

(Filta de conocimicnto)

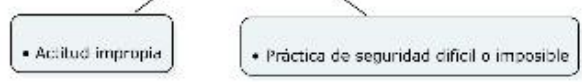

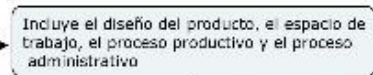

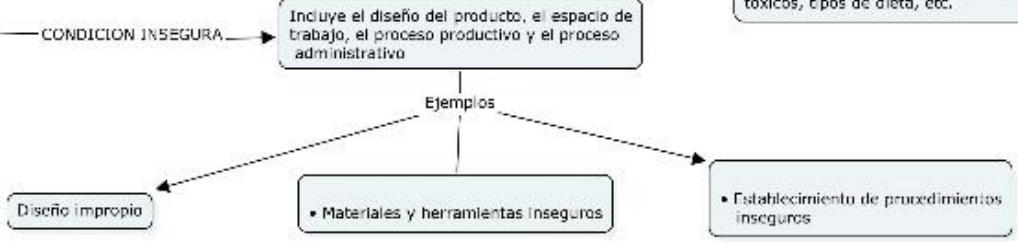




\section{Referencias}

Gabriel García Acosta.La ergonomía desde la visión sistémica (2002)

Ramírez Cavassa César. Seguridad

Industrial. México. Ed. Limusa (1990 ) 
Publicación semestral No. 11 (2019) 57-62 
vel convallis posuere augue conubia primis fusce. Diam fringilla mi malesuada sagittis nisl metus pharetra justo habitant a dictum mollis, potenti proin nisi viverra ultrices libero congue dignissim laoreet nostra neque. 3-5

Lorem ipsum dolor sit amet consectetur adipiscing elit volutpat, ultricies in primis mollis enim lobortis morbi sodales, praesent nisi nec cubilia integer congue feugiat. Quis at blandit erat nisl lacus turpis vehicula penatibus integer tristique felis taciti, natoque praesent congue morbi vel convallis posuere augue conubia primis fusce. Diam fringilla mi malesuada sagittis nisl metus pharetra justo habitant a dictum mollis, potenti proin nisi viverra ultrices libero congue dignissim laoreet nostra neque. 6,7

Lorem ipsum dolor sit amet consectetur adipiscing elit volutpat, ultricies in primis mollis enim lobortis morbi sodales, praesent nisi nec cubilia integer congue feugiat. Quis at blandit erat nisl lacus turpis vehicula penatibus integer tristique felis taciti, natoque praesent congue morbi vel convallis posuere augue conubia primis fusce. Diam fringilla mi malesuada sagittis nisl metus pharetra justo habitant a dictum mollis, potenti proin nisi viverra ultrices libero congue dignissim laoreet nostra neque. 8

\section{Lorem ipsum dol}

Lorem ipsum dolor sit amet consectetur adipiscing elit volutpat, ultricies in primis mollis enim lobortis morbi sodales, praesent nisi nec cubilia integer congue feugiat. Quis at blandit erat nisl lacus turpis vehicula penatibus integer tristique felis taciti, natoque praesent congue morbi vel convallis posuere augue conubia primis fusce. Diam fringilla mi malesuada sagittis nisl metus pharetra justo habitant a dictum mollis, potenti proin nisi viverra ultrices libero congue dignissim laoreet nostra neque. 9

\section{Figure 1. Lorem ipsum dolor sit amet consectetur adipiscing elit volutpat}

Lorem ipsum dolor_sit_amet consectetur adipiscing elit volutpat, ultricies in primis mollis enim lobortis morbi

Lorem ipsum dolor sit amet consectetur adipiscing elit volutpat, ultricies in primis mollis enim lobortis morbi sodales, praesent nisi nec cubilia integer congue feugiat. Quis at blandit erat nisl lacus turpis vehicula penatibus integer tristique felis taciti, natoque praesent congue morbi vel convallis posuere augue conubia primis fusce. Diam fringilla mi malesuada sagittis nisl metus pharetra justo habitant a dictum mollis, potenti proin nisi viverra ultrices libero congue dignissim laoreet nostra neque. 1,2

Lorem ipsum dolor sit amet consectetur adipiscing elit volutpat, ultricies in primis mollis enim lobortis morbi sodales, praesent nisi nec cubilia integer congue feugiat. Quis at blandit erat nisl lacus turpis vehicula penatibus integer tristique felis taciti, natoque praesent congue morbi
Lorem ipsum dolor sit amet consectetur adipiscing elit volutpat, ultricies in primis mollis enim lobortis morbi sodales, praesent nisi nec cubilia integer congue feugiat. Quis at blandit erat nisl lacus turpis vehicula penatibus integer tristique felis taciti, natoque praesent congue morbi vel convallis posuere augue conubia primis fusce. Diam fringilla mi malesuada sagittis nisl metus pharetra justo habitant a dictum mollis, potenti proin nisi viverra ultrices libero congue dignissim laoreet nostra neque. 
Lorem ipsum dolor sit amet consectetur adipiscing elit volutpat, ultricies in primis mollis enim lobortis morbi sodales, praesent nisi nec cubilia integer congue feugiat.

\section{Referencias}

[1] Dilla T, Valladares A, Lizán L, Sacristán JA. Adherencia y persistencia terapéutica: causas, consecuencias y estrategias de mejora. Aten. Primaria 2009; 41(6): 342-48.

[2] Silva G, Galeano E, Correa J. Compliance with the treatment. Acta Médica Colombiana 2005; 30(4): 268-73.

[3] Viesca TC. Paternalismo médico y consentimiento informado. En Márquez-Romero R, Rocha- Cacho WV, editors. Consentimiento informado. Fundamentos y problemas de su aplicación práctica. 1st ed. México: UNAM; 2017: 1-22.

[4] Grady P, Lucio-Gough L. El automanejo de las enfermedades crónicas un método integral de atención. Panam. J. Public. Health. 2015; 35(9): 187-194.

[5] Cañete-Villafranca R, Guilhem D, Brito-Pérez K. Paternalismo médico. Rev. Med. Elect. 2013; 35(2): 144-152.

[6] Caballo C, Pascual A. Entrenamiento en habilidades de automanejo en personas con enfermedades crónicas: un estudio cuasi-experimental. En: Universidad de Salamanca, editor. IX Jornadas Científicas Internacionales de investigación sobre personas con discapacidad. Salamanca; INICO; 2012: 1-12.

[7] Millaruelo-Trillo JM. Importancia de la implicación del paciente en el autocontrol de su enfermedad: paciente experto. Importancia de las nuevas tecnologías como soporte al paciente autónomo. Aten. Primaria 2010; 42(1): 41-47.

[8] Bonal-Ruiz R, Cascaret-Soto X. Reflexión y debate ¿Automanejo, autocuidado o autocontrol en enfermedades crónicas? Acercamiento a su análisis e interpretación. MEDISAN. 2009; 13(1): 1-10.

[9] Lorig K, Ritter PL, Villa F, Piette JD. Spanish diabetes self-management with and without automated telephone reinforcement. Diabetes Care 2008; 31(3): 408-14.

[10] Kidney Disease Improved Global Outcomes (KDIGO). KDIGO 2012 Clinical Practice Guideline for the Evaluation and Management of Chronic Kidney Disease. Off. J. Int. Soc. Nephrol. 2013; 3(1): 1-163. 